\title{
Dissemination and Molecular Epidemiology of KPC-Producing Klebsiella pneumoniae Collected in Puerto Rico Medical Center Hospitals during a 1-Year Period
}

\author{
Iraida E. Robledo, ${ }^{1}$ Guillermo J. Vázquez, ${ }^{1}$ Ellen S. Moland, ${ }^{2}$ Edna E. Aquino, ${ }^{1}$ \\ Richard V. Goering, ${ }^{2}$ Kenneth S. Thomson, ${ }^{2}$ María I. Santé, ${ }^{3}$ and Nancy D. Hanson ${ }^{2}$ \\ ${ }^{1}$ Department of Microbiology and Medical Zoology, School of Medicine, University of Puerto Rico, P.O. Box 365067, San Juan, \\ PR 00936-5067, USA \\ ${ }^{2}$ Department of Medical Microbiology, School of Medicine, Creighton University, Omaha, NE 68178, USA \\ ${ }^{3}$ Department of Pathology and Laboratory Medicine, School of Medicine, University of Puerto Rico, San Juan, PR 00936-5067, USA
}

Correspondence should be addressed to Iraida E. Robledo, iraida.robledo@upr.edu

Received 25 August 2011; Revised 29 September 2011; Accepted 3 October 2011

Academic Editor: H. T. Sørensen

Copyright ( 2011 Iraida E. Robledo et al. This is an open access article distributed under the Creative Commons Attribution License, which permits unrestricted use, distribution, and reproduction in any medium, provided the original work is properly cited.

During a 2003-2004 PCR-based surveillance study conducted in 6 Puerto Rico Medical Center hospitals, 27/92 multi-beta-lactamresistant Klebsiella pneumoniae strains were identified as carbapenemase (KPC) positive in 4 hospitals. The objectives of this study were to identify the KPC variants, their genetic relatedness, and any other beta-lactamases present. Susceptibility testing, pulsed field gel electrophoresis (PFGE), isoelectric focusing, PCR, and DNA sequencing were performed. KPC variants -2 , -3 , -4 , and -6 were identified. Additional beta-lactamases detected were TEM, DHA, OXA-9 and -30. Antimicrobial susceptibility to carbapenems varied depending on the KPC variant. Five PFGE genetically related groups were identified in 15 isolates and 12 unrelated types. PFGE profiles suggested that both clonal and horizontal transfer are contributing to the dissemination of these isolates among the various hospitals. Comparison of the 2003 and a 2009 surveillance studies showed a significant increase in the KPC-positive K. pneumoniae isolates in the latter.

\section{Introduction}

The detection of carbapenemase producing isolates is a major clinical concern since carbapenems are the drug of choice for the treatment of infections caused by multidrugresistant Gram-negative bacteria. KPC stands for Klebsiella pneumoniae carbapenemase, which are classified as Bush subgroup $2 \mathrm{f}$ Class A serine-based enzymes, inhibited by clavulanic acid and tazobactam. They are capable of hydrolyzing beta-lactam antibiotics of all classes [1-3]. KPCproducing isolates, which were initially restricted to hospitals located in the northeastern USA, have recently been detected in other USA regions and in other countries worldwide [1-3]. They have been identified in K. pneumoniae, other Enterobacteriaceae, and recently in Pseudomonas aeruginosa, and Acinetobacter baumannii [1-9]. Serious nosocomial outbreaks have been associated with these KPC-positive organisms [10-14]. This carbapenemase-encoding gene is found on transferable plasmids associated with transposon Tn4401 $[15,16]$. Ten KPC variants (KPC-2 to -11 ) have been described so far (http://www.lahey.org/studies/) differing between them in 1- or 2-point mutation [1-3]. KPCproducing isolates may be difficult to detect since elevated carbapenem MIC are not always evident $[2,17]$. This poses a major therapeutic challenge, as treatment may be inadequate and therefore may lead to significant increase in patients' mortality, morbidity, and hospitalization costs. To improve the detection of KPC-positive Enterobacteriaceae, the Clinical and Laboratory Standards Institute (CLSI) in June 2010 changed the carbapenem (imipenem, doripenem, and meropenem) MIC susceptible breakpoints from $\leq 4$ to $\leq 1 \mu \mathrm{g} / \mathrm{mL}$, and for ertapenem from $\leq 2$ to $\leq 0.25 \mu \mathrm{g} / \mathrm{mL}$ [18]. 
A retrospective antimicrobial resistance study performed at the Puerto Rico Medical Center suggested the presence of broad-spectrum beta-lactamases as one of the causes of the resistance problem [19]. During a 2003-2004 multibeta-lactam resistance surveillance study performed in this medical center, 92 of 285 (32\%) unique and consecutive clinical isolates of K. pneumoniae were identified as multi-betalactam resistant. In this study, multi-beta-lactam resistance was defined as resistance to any of the carbapenems and/or two or more of the following antibiotics: ceftriaxone, cefotaxime, ceftazidime, cefepime, aztreonam, and piperacillintazobactam. PCR screening for the presence of familyspecific $\beta$-lactamase genes detected the KPC gene in 27 of the 92 multi-beta-lactam resistant $(29 \%)$ or $10 \%$ of the $285 \mathrm{~K}$. pneumoniae isolates. The objectives of this study were to identify the KPC variants, their genetic relatedness, and the presence of other $\beta$-lactamases in the $27 \mathrm{~K}$. pneumoniae clinical isolates.

\section{Materials and Methods}

2.1. Description of Hospitals Facilities. The Puerto Rico Medical Center is located in the San Juan Metropolitan Area. It includes six hospitals and/or specialized units with a total bed capacity of 858 , a central clinical laboratory facility and the University of Puerto Rico, Medical Sciences Campus. Within a 10 mile radius, there are 10 private or community hospitals where many of the faculty also have admitting privileges.

2.2. Bacterial Isolates. The $27 \mathrm{KPC}$-positive $K$. pneumoniae strains isolated in 2003-2004 were identified and screened for inclusion in the study at the Puerto Rico Medical Center clinical laboratory utilizing the VITEK DCS-R5 System (bioMérieux Inc., Hazelwood, MO, USA). The following information was obtained from the bacteriology laboratory report: the in vitro antimicrobial susceptibilities, patient's age, sex, anatomical site of infection, hospital, and hospital ward. No attempts were made to evaluate patients' therapies or clinical outcomes.

2.3. Susceptibility Testing. Antimicrobial susceptibilities were determined by using commercial microdilution MIC panels (TREK Diagnostic System, Inc. Cleveland, OH, USA) following the manufacturer's instruction. The recent CLSI carbapenem susceptibility breakpoint changes for Enterobacteriaceae were utilized [18]. All isolates with an intermediate susceptibility to the antibiotics were considered resistant.

2.4. $\beta$-Lactamase PCR Screening. The presence of familyspecific beta-lactamase genes were identified by PCR using panels of specific primers for the detection of the following genes: (1) plasmid-encoding AmpC $\beta$-lactamases (MOX, CMY, LAT, BIL, DHA, ACC, MIR, ACT, and FOX), (2) extended spectrum beta-lactamases (CTX-M groups I, II, III, and IV and TEM), (3) carbapenemase-hydrolyzing enzymes (KPC), and (4) metallo- $\beta$-lactamases (IMP and VIM). The oxacillinases OXA-30 and OXA-9 were used to try to identify some of the bands detected by isoelectric focusing (see below). The SHV-1 $\beta$-lactamase is chromosomally encoded in the majority of isolates of K. pneumonia; therefore, SHV primer was not used on the $K$. pneumoniae isolates. The bacterial DNA template, primers used, and the PCR conditions were performed as described previously [9, 20-22].

2.5. Isoelectric Focusing (IEF). IEF of sonicated crude cell extracts of the $27 \mathrm{KPC}$-positive isolates was performed as previously described $[20,23]$ to determine the number of betalactamases and their isoelectric points (pIs), the capabilities of cloxacillin and clavulanate to inhibit the beta-lactamases, and the capability of the $\beta$-lactamase(s) to hydrolyze cefotaxime or imipenem.

2.6. DNA Sequencing Analysis. PCR amplification of KPC full-length gene products was sequenced with primers KPCF1 and KPCR1, which flank the gene [9]. All PCR amplicons generated were sequenced independently and bidirectionally at least twice. Sequence alignment and analysis were performed online using the BLAST program (http:// www.ncbi.nlm.nih.gov/). PCR products were sequenced at the Creighton University Molecular Biology Core Facility using an ABI Prism 3100 Avant genetic analyzer (Applied Biosystems, Foster City, CA, USA).

2.7. Pulsed Field Gel Electrophoresis (PFGE). To determine their genetic relatedness, the $27 \mathrm{~K}$. pneumoniae isolates were analyzed by PFGE. For each isolate, DNA was prepared by in situ lysis of cells encased in agarose plugs, and digested with $X b a \mathrm{I}$ as previously described $[24,25]$. PFGE was performed using a Bio-Rad CHEF DR III System at $6 \mathrm{~V} / \mathrm{cm}, 14^{\circ} \mathrm{C}$, $120^{\circ} \mathrm{C}$ included angle, with switching from 5 to $15 \mathrm{~s}$ for $10 \mathrm{~h}$, followed by switching from 15 to $60 \mathrm{~s}$ for $13 \mathrm{~h}$. Images of ethidium-bromide stained gels were archived using a BioRad Gel Doc 1000 System. PFGE profiles were compared using BioNumerics $\mathrm{v}$ 5.1. Isolates with $85 \%$ or greater similarity in the bands patterns were assigned to the same PFGE groups.

2.8. Comparison between 2003 and 2009 Surveillance Periods. A comparison between the total number of multi-betalactam-resistant and KPC-positive K. pneumoniae collected from the Puerto Rico Medical Center for similar 6-month surveillance period for years 2003 (present study) and a recently published 2009 surveillance study (7) was performed.

2.9. Statistical Analyses. The Two-tailed Fisher exact test or the Mixed Model Logistic Regression analyses were utilized to detect significant differences. A $P$ value $\leq 0.05$ was considered statistically significant.

\section{Results}

The $27 \mathrm{~K}$. pneumoniae strains were isolated from four of the six Puerto Rico Medical Center hospitals (MC1, MC2, MC3, and MC4 and an unidentified hospital) with a total capacity of 629 beds. The types of clinical services offered by these hospitals include medicine, pediatrics, surgery, and trauma. 
TABLE 1: Baseline epidemiological data of the patients with KPC-positive K. pneumoniae isolates.

\begin{tabular}{lccccccc}
\hline \multirow{2}{*}{ Hospital } & \multirow{2}{*}{ No. of beds } & \multicolumn{2}{c}{ Gender $^{\mathrm{c}}$} & \multicolumn{4}{c}{ Anatomical site of infection } \\
& & $\mathrm{F}$ & $\mathrm{M}$ & Blood & Sputum & Urine & Misc. $^{\text {d }}$ \\
\hline MC1 & 262 & 8 & 5 & 4 & 2 & 2 & 5 \\
MC2 & 155 & 0 & 3 & 0 & 0 & 2 & 1 \\
MC3 & 150 & 4 & 4 & 3 & 2 & 0 & 3 \\
MC4 & 62 & 0 & 2 & 1 & 1 & 0 & 0 \\
NR $^{\mathrm{a}}$ & NA $^{\mathrm{b}}$ & 1 & 0 & 0 & 0 & 1 & 0 \\
\hline Total & 629 & 13 & 14 & 8 & 5 & 5 & 9 \\
\hline
\end{tabular}

${ }^{\mathrm{a}}$ NR: Unidentified hospital (not reported).

${ }^{b} \mathrm{NA}$ : no. of applicable.

${ }^{\mathrm{c}} \mathrm{F}$ : female; M: male.

${ }^{\mathrm{d}}$ Misc.: miscellaneous.

Table 1 shows the baseline epidemiological information of the patients with KPC-positive $K$. pneumoniae isolates. Twenty-one of 27 isolates were obtained from either hospital MC1 or MC3. A similar distribution of KPC-positive isolates was noted between females and males patients or the anatomical site of infection.

Table 2 shows the KPC variants, isoelectric point (pI) value, and other beta-lactamases identified in the 27 isolates. Four KPC variants were identified: KPC-2 in 8 isolates, $\mathrm{KPC}-3$ in 14 isolates, KPC-4 in 4 isolates, and the novel KPC-6 (GenBank accession number EU555534.1) in one isolate. The DNA sequence of this new variant showed a single point mutation at position 239 where a valine was substituted for a glycine. The IEF results indicated two pI values consistent with a KPC-like enzyme, a band with a $\mathrm{pI}$ of 7.65 identified as KPC- 4 and a pI value of 6.7 for the rest of the variants including KPC- 6 . The pIs of the other betalactamases detected in the isolates ranged from 5.25 to 8.2, with the number of beta-lactamase bands per isolate varying from 2 to 7. Other beta-lactamases genes identified by PCR were the plasmid-encoded DHA-type AmpC in 4 isolates, OXA-30 in 5 isolates, OXA-9 in 6 isolates, and TEM in $26 / 27$ isolates. None of the TEM beta-lactamases hydrolyzed cefotaxime on IEF and were not further characterized.

Table 3 shows the in vitro susceptibility test results to selected antibiotics. The susceptibility to beta-lactam antibiotics demonstrated that $59 \%$ of the isolates were susceptible to cefepime followed by cefoxitin (37\%), meropenem (37\%), and imipenem (33\%). None of the isolates were susceptible to ertapenem, ceftazidime, or aztreonam. For the non-betalactam antibiotics, the organisms were consistently susceptible to tigecycline (100\%) and amikacin (89\%). Susceptibility to polymyxins was not determined.

Table 4 shows the susceptibility to carbapenems according to the KPC variant present in the K. pneumoniae isolates. All isolates were resistant to ertapenem irrespective of the $\mathrm{KPC}$ variant. Isolates with $\mathrm{KPC}-2$ and -6 were resistant to all the carbapenem tested. The four isolates with KPC-4 were susceptible to imipenem and meropenem, while those with KPC-3 demonstrated variable susceptibility.

Figure 1 shows the dendrogram and PFGE results to determine the genetic relatedness of the $27 \mathrm{KPC}$-positive $\mathrm{K}$. pneumoniae isolates. A total of 17 distinct PFGE groups were
TABLE 2: KPC variants and other beta-lactamases in the $27 \mathrm{~K}$. pneumoniae isolates.

\begin{tabular}{lccc}
\hline $\begin{array}{l}\text { KPC variant by } \\
\begin{array}{l}\text { DNA sequencing } \\
\text { (total isolates) }\end{array}\end{array}$ & pI of KPC & $\begin{array}{c}\text { Distribution } \\
\text { isolates per } \\
\text { enzymes }\end{array}$ & $\begin{array}{c}\text { Others } \\
\text { beta-lactamases } \\
\text { identified by PCR }\end{array}$ \\
\hline KPC-2 (8) & 6.7 & 4 & OXA-30, TEM, DHA \\
& & 4 & TEM \\
KPC-3 (14) & 6.7 & 1 & OXA-9, TEM \\
& & 8 & OXA-9 \\
KPC-4 (4) & 7.65 & 4 & TEM \\
KPC-6 (1) & 6.7 & 1 & TEM \\
\hline
\end{tabular}

TABle 3: MIC-50, MIC-90, and \% susceptible of 27 KPC-producing K. pneumoniae Isolates.

\begin{tabular}{lcccc}
\hline Test agent & $\begin{array}{c}\text { Range } \\
(\mu \mathrm{g} / \mathrm{mL})\end{array}$ & $\begin{array}{c}\mathrm{MIC}_{50} \\
(\mu \mathrm{g} / \mathrm{mL})\end{array}$ & $\begin{array}{c}\mathrm{MIC}_{90} \\
(\mu \mathrm{g} / \mathrm{mL})\end{array}$ & $\begin{array}{c}\text { \% susceptible } \\
\text { (no. of suscep- } \\
\text { tible/no. of } \\
\text { tested isolates })\end{array}$ \\
\hline $\begin{array}{l}\text { Piperacillin/ } \\
\text { tazobactam }\end{array}$ & $32 / 4->128 / 4$ & $>128 / 4$ & $>128 / 4$ & 0 \\
Cefoxitin & $\leq 4->64$ & 16 & $>64$ & $37(10 / 27)$ \\
Ceftazidime & $16->128$ & 32 & 128 & 0 \\
Ceftriaxone & $4->128$ & 32 & $>128$ & $11(3 / 27)$ \\
Cefepime & $\leq 4->32$ & $\leq 4$ & 32 & $59(16 / 27)$ \\
Aztreonam & $16->32$ & $>32$ & $>32$ & 0 \\
Ertapenem & $\leq 2->16$ & $\leq 2$ & $>16$ & 0 \\
Imipenem & $\leq 0.5->16$ & 2 & 16 & $33(9 / 27)$ \\
Meropenem & $\leq 1->8$ & 4 & $>8$ & $37(10 / 27)$ \\
Trimethoprim/ & $\leq 0.5->4$ & $>4$ & $>4$ & $26(7 / 27)$ \\
sulfamethoxazole & & & & $33(9 / 27)$ \\
Ciprofloxacin & $\leq 0.5->4$ & $>4$ & $>4$ & $30(8 / 27)$ \\
Gentamicin & $\leq 2->16$ & 8 & $>16$ & $89(24 / 27)$ \\
Amikacin & $\leq 8-32$ & $\leq 8$ & 16 & $\leq 1$ \\
Tigecycline & $\leq 1-4$ & $\leq 1$ & $100(27 / 27)$ \\
\hline
\end{tabular}




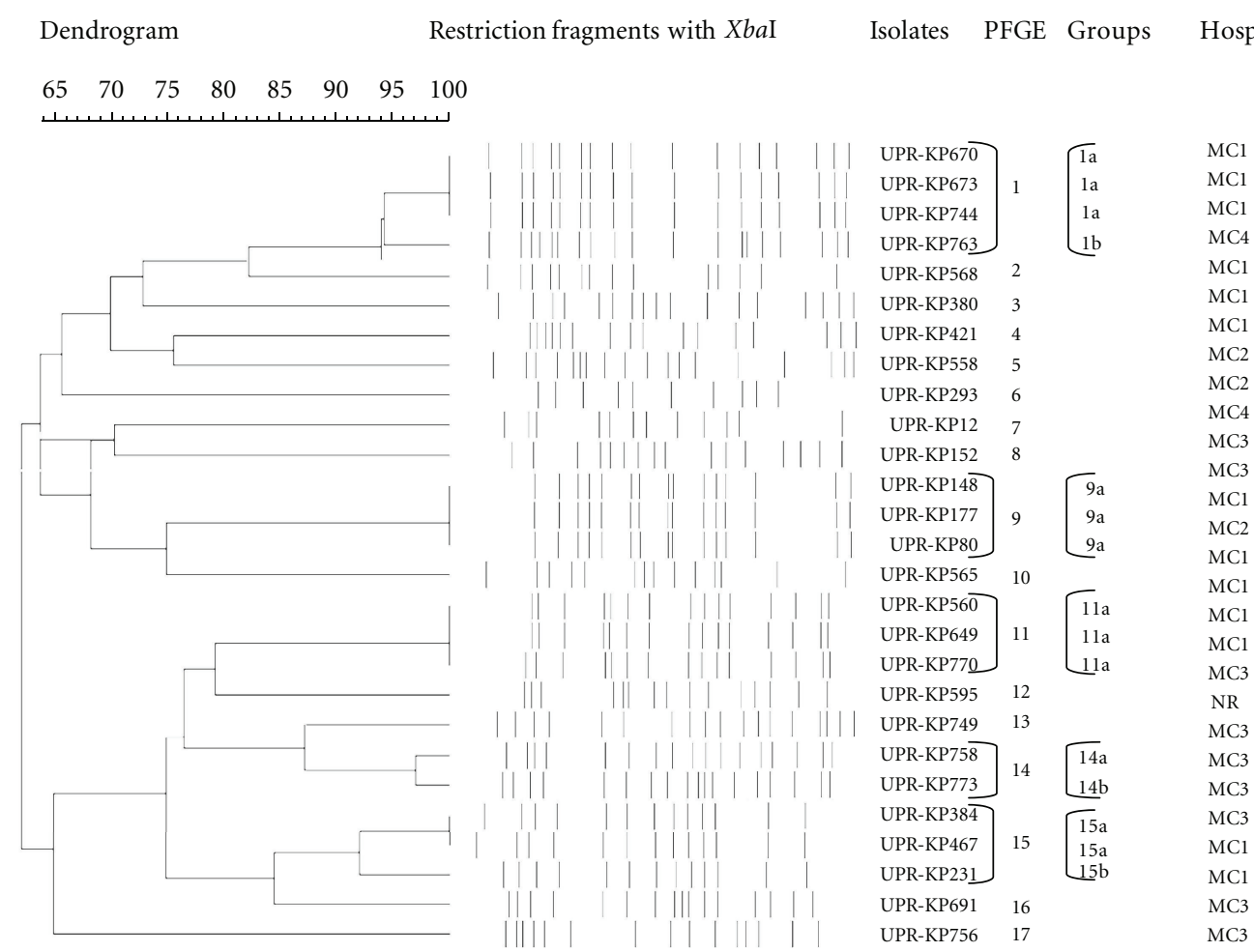

FIGURE 1: Genetic relatedness: PFGE for the 27 KPC-positive K. pneumoniae isolates.

TABLE 4: Total number and \% of susceptibility to carbapenems according to KPC variant.

\begin{tabular}{|c|c|c|c|c|}
\hline \multirow{2}{*}{$\begin{array}{l}\text { Type of } \\
\text { KPC } \\
\text { variant }\end{array}$} & \multirow{2}{*}{$\begin{array}{l}\text { Number of } \\
\text { isolates }\end{array}$} & \multicolumn{3}{|c|}{$\begin{array}{c}\% \text { susceptible } \\
\text { (no. of susceptible/no. of tested isolates) }\end{array}$} \\
\hline & & Ertapenem & Imipenem & Meropenem \\
\hline KPC-2 & 8 & $0(0 / 8)$ & $0(0 / 8)$ & $0(0 / 8)$ \\
\hline KPC-3 & 14 & $0(0 / 14)$ & $29(4 / 14)$ & $43(6 / 14)$ \\
\hline KPC-4 & 4 & $0(0 / 4)$ & $100(4 / 4)$ & $100(4 / 4)$ \\
\hline KPC-6 & 1 & $0(0 / 1)$ & $0(0 / 1)$ & $0(0 / 1)$ \\
\hline
\end{tabular}

identified. Fifteen of the isolates were distributed between five distinct groups, and the remaining 12 isolates were unrelated to each other.

Table 5 describes the characterization and distribution of the $15 \mathrm{~K}$. pneumoniae isolates in the 5 PFGE-related groups. All isolates belonging to a related group possessed the same beta-lactamases and KPC variant. Hospital MC1 had 4/5 of the related groups followed by Hospital MC3 with 3/5. The other isolates from the remaining 3 groups were spread among the 4 hospitals, most of them distributed between Hospitals MC1 and MC3, while Hospital MC2 and MC4 with 1 related group each. Two hospitals each had a single unique related group, Hospital MC1 with group 11 and Hospital MC3 with group 14. Six of 11 (54\%) of the related isolates were detected in the intensive care units (ICUs). Five of them were identified in Hospital MC1, representing 3/5 related groups. The distributions were as follows: 2 isolates from group 1, two from group 9, and two from group 11.

Table 6 describes the distribution and characterization of the 12 PFGE-unrelated K. pneumoniae isolates. KPC variants and other beta-lactamases were also identified in these isolates. Hospitals MC1 and MC3 had most of the 12 unrelated isolates, with 4 isolates each, Hospital MC2 had 2, Hospital MC4 and the unidentified hospital 1 each. Eighty-three percent of these unrelated $K$. pneumoniae were identified in general hospitals wards and only 2 or $17 \%$ in the intensive care units. The difference in the distribution in either the ICU or general ward of the 5 PFGE-related groups and the 12 unrelated isolates was statistically significant $(P \leq 0.05)$.

Table 7 shows the distribution of isolates in the four hospitals during the 1-year surveillance period according to related and unrelated PFGE groups and type of KPC variants. Hospitals MC1 and MC3 had the highest number of KPCpositive isolates with $13 / 27$ and $8 / 27$, respectively. KPC-2 and -3 were the most common variants observed and detected in all the hospitals. The KPC-2 variant was detected in PFGErelated group 15 and in five unrelated isolates (groups 2, 6, 7,8 , and 16). KPC-3 was identified in three related groups $(1,11$, and 14$)$ and in five unrelated isolates (groups $3,5,12$, 13 , and 17). KPC-4 was detected in related group 9 for two consecutive months in hospitals MC1, MC2, and MC3, and four months later, that same KPC variant was detected in a PFGE-unrelated isolate (group 10) in Hospital MC1. KPC-6 was identified once in hospital MC1 six months after starting the study. The movement of genetically related isolates into different hospitals was noted with three PFGE groups. PFGE 
TABLE 5: Distribution and characterization of the five K. pneumoniae PFGE-related groups (15/27).

\begin{tabular}{|c|c|c|c|c|c|c|c|}
\hline Isolates & \multicolumn{2}{|c|}{ PFGE groups } & $\begin{array}{c}\text { KPC } \\
\text { variant }\end{array}$ & Other beta-lactamases & $\begin{array}{l}\text { Anatomical } \\
\text { site }\end{array}$ & Hospital & $\begin{array}{c}\text { Hospital } \\
\text { unit }^{\mathrm{b}}\end{array}$ \\
\hline UPR-Kp670 & \multirow{4}{*}{1} & $1 \mathrm{a}$ & \multirow{4}{*}{ KPC-3 } & \multirow{4}{*}{ TEM,OXA-9 } & Urine & $\mathrm{MC1}$ & ICU \\
\hline UPR-Kp673 & & 1a & & & Misc. & $\mathrm{MC1}$ & Gen Ward \\
\hline UPR-Kp744 & & la & & & Blood & $\mathrm{MC1}$ & $\mathrm{ICU}$ \\
\hline UPR-Kp763 & & $1 \mathrm{~b}$ & & & Blood & MC4 & Other \\
\hline UPR-Kp177 & \multirow{3}{*}{9} & $9 a$ & \multirow{3}{*}{ KPC-4 } & \multirow{3}{*}{ TEM } & Misc. $^{\text {a }}$ & $\mathrm{MC1}$ & $\mathrm{ICU}$ \\
\hline UPR-Kp080 & & $9 \mathrm{a}$ & & & Urine & MC2 & $\mathrm{ICU}$ \\
\hline UPR-Kp148 & & $9 \mathrm{a}$ & & & Blood & MC3 & Gen Ward \\
\hline UPR-Kp770 & \multirow{3}{*}{11} & $11 \mathrm{a}$ & \multirow{3}{*}{ KPC-3 } & \multirow{3}{*}{ TEM } & Blood & $\mathrm{MC1}$ & $\mathrm{ICU}$ \\
\hline UPR-Kp649 & & $11 \mathrm{a}$ & & & Misc. & MC1 & Gen Ward \\
\hline UPR-Kp560 & & $11 \mathrm{a}$ & & & Sputum & $\mathrm{MC1}$ & ICU \\
\hline UPR-Kp758 & \multirow[b]{2}{*}{14} & $14 \mathrm{a}$ & \multirow[b]{2}{*}{ KPC-3 } & \multirow[b]{2}{*}{ TEM } & Blood & MC3 & Other \\
\hline UPR-Kp773 & & $14 \mathrm{~b}$ & & & Sputum & MC3 & Other \\
\hline UPR-Kp231 & \multirow{3}{*}{15} & $15 b$ & \multirow{3}{*}{ KPC-2 } & \multirow{3}{*}{ DHA,TEM,OXA-30 } & Misc. & $\mathrm{MC1}$ & Gen Ward \\
\hline UPR-Kp467 & & $15 \mathrm{a}$ & & & Sputum & $\mathrm{MC1}$ & Gen Ward \\
\hline UPR-Kp384 & & $15 \mathrm{a}$ & & & Misc. & MC3 & Gen Ward \\
\hline
\end{tabular}

${ }^{a}$ Misc.: miscellaneous.

${ }^{\mathrm{b}}$ Gen Ward: General Ward; ICU: Intensive Care Unit; other: site unspecified.

TABLE 6: Distribution and characterization of the 12 PFGE-unrelated K. pneumoniae isolates.

\begin{tabular}{|c|c|c|c|c|c|c|}
\hline Isolates & PFGE groups & KPC variant & Other beta-lactamases & Anatomical site & Hospital & Hospital unit $^{\mathrm{c}}$ \\
\hline UPR-Kp421 & 4 & KPC-6 & TEM, OXA-30 & Blood & $\mathrm{MC1}$ & Gen Ward \\
\hline UPR-Kp691 & 16 & KPC-2 & DHA, TEM, OXA-30 & Sputum & MC3 & Gen Ward \\
\hline UPR-Kp012 & 7 & KPC-2 & TEM & Sputum & MC4 & ICU \\
\hline UPR-Kp152 & 8 & KPC-2 & TEM & Misc. $^{\text {a }}$ & MC3 & Gen Ward \\
\hline UPR-Kp293 & 6 & KPC-2 & TEM & Urine & MC2 & Gen Ward \\
\hline UPR-Kp568 & 2 & KPC-2 & TEM & Misc. & $\mathrm{MC1}$ & Gen Ward \\
\hline UPR-Kp558 & 5 & KPC-3 & OXA-9 & Misc. & MC2 & Gen Ward \\
\hline UPR-Kp749 & 13 & KPC-3 & TEM, OXA-9 & Urine & $\mathrm{NR}^{\mathrm{b}}$ & Other \\
\hline UPR-Kp380 & 3 & KPC-3 & TEM & Blood & $\mathrm{MC1}$ & Gen Ward \\
\hline UPR-Kp756 & 17 & $\mathrm{KPC}-3$ & TEM & Blood & MC3 & Gen Ward \\
\hline UPR-Kp595 & 12 & KPC-3 & TEM & Misc. & MC3 & Gen Ward \\
\hline UPR-Kp565 & 10 & KPC-4 & TEM & Urine & $\mathrm{MC1}$ & ICU \\
\hline
\end{tabular}

${ }^{a}$ Misc.: miscellaneous.

${ }^{b}$ NR: unidentified hospital (not reported).

${ }^{\mathrm{c}}$ Gen Ward: General Ward; ICU: Intensive Care Unit; other: site unspecified.

TABle 7: Distribution of the 27 K. pneumoniae isolates by hospital, PFGE Groups ${ }^{\mathrm{a}}$, and KPC variants ${ }^{\mathrm{b}}$ during the 1 -year surveillance study period.

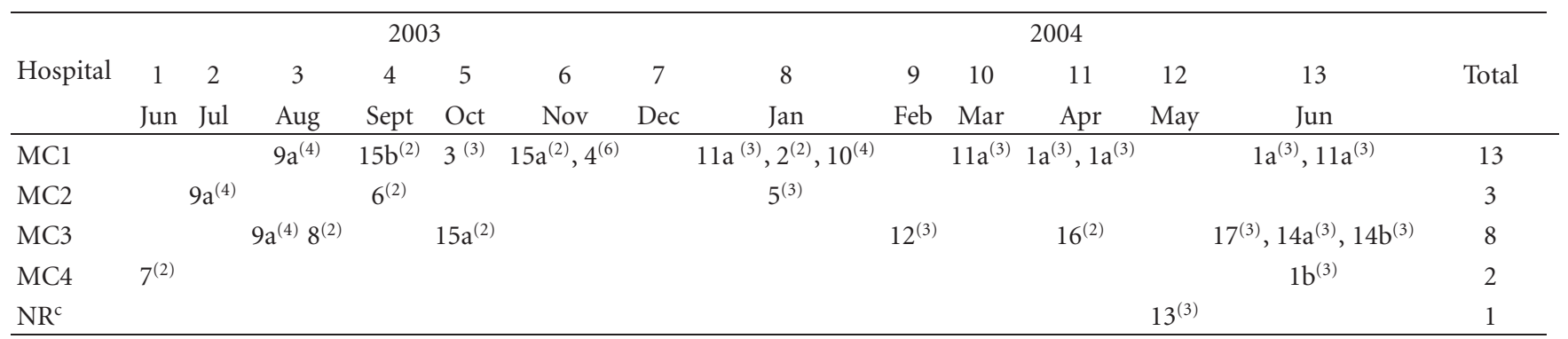

${ }^{a}$ Numbers followed by a letter represent the related PFGE groups $(1,9,11,14$, and 15$)$ and numbers alone represent the unrelated PFGE groups $(2,3,4,5,6$, $7,8,10,12,13,16$, and 17$)$.

${ }^{\mathrm{b}}$ Type of KPC variant found in the isolates is shown in the superscript: KPC- $2^{(2)}, \mathrm{KPC}-3^{(3)}, \mathrm{KPC}-4^{(4)}$, and $\mathrm{KPC}-6^{(6)}$.

${ }^{\mathrm{c}}$ NR: unidentified hospital (not reported). 
group 9 was detected in Hospital MC2 in July, 2003, and the following month was identified in Hospitals MC1 and MC3. In September and November, 2003, group 15 was detected in Hospital MC1 and in October, 2003 in Hospital MC3. Finally, group 1 detected in Hospital MC1 in April 2004 was identified in Hospital MC4 in June, 2004.

A statistical comparison of the number of KPC-positive isolates identified within the multi-beta-lactam-resistant $K$. pneumonia isolated during similar six-month periods for the years 2003 and 2009 is shown in Table 8. The results showed a statistically significant increase in the number of multibeta-lactam-resistant and KPC-positive K. pneumoniae in the four hospitals during the 2009 surveillance when compared to the present study $(52 / 11,21 \%$ versus $109 / 92,84 \%$; $P$ value $\leq 0.01)$.

\section{Discussion}

This paper represents the first molecular surveillance study of KPC and other beta-lactamases for K. pneumoniae strains at the Puerto Rico Medical Center hospitals. It is important to recognize that patients admitted to the PRMC represent, for the most part, referrals from all over the island of complicated or seriously ill patients. Most of these patients have been previously hospitalized at other institutions and subjected to different therapies including parenteral antibiotics. Because of the complicated nature of their conditions, many patients admitted have prolonged hospitalizations, several invasive procedures, and extensive antibiotic treatments. All these factors may contribute to the patients' acquisition of multidrug-resistant bacteria.

Four KPC variants were detected but, as expected, KPC2 and KPC-3 were the most prevalent [1]. KPC-4 with a pI value of 7.65 was identified in 4 isolates belonging to PFGE groups 9 and 10. This variant, initially detected in Enterobacter sp. from Scotland [26] is, to our knowledge, identified for the first time in K. pneumoniae. A new variant, KPC-6, was identified in one isolate. The detection of multiple KPC variants in our isolates may represent random mutational events or introduction of the genes from external sources. Additional beta-lactamases identified in these isolates are OXA-30, OXA-9, TEM, and plasmid-encoded AmpC-DHA. The presence of multiple beta-lactamases in KPC-positive isolates has been previously reported $[1,3,14,27]$.

The in vitro antimicrobial resistance to the beta-lactam antibiotics, fluoroquinolones, trimethoprim sulfa, and gentamicin was observed suggesting the presence of multiple mechanisms of antibiotics resistance. Only amikacin and tigecycline demonstrated good to excellent in vitro antimicrobial activity against these isolates; unfortunately, susceptibility to the polymyxins were not performed. These data are in agreement with previous reports showing the limited therapeutic options available for the treatment of infections caused by KPC-positive bacteria [1, 28].

All K. pneumoniae isolates were resistant to ertapenem, but nearly one-third were susceptible to imipenem and/or meropenem, even though the recent CLSI carbapenem breakpoints were used [18]. Carbapenem susceptible isolates
TABLE 8: Comparison of multi-beta-lactam-resistant and KPCPositive K. pneumoniae for similar 6-month surveillance periods for years 2003 and 2009 at the Puerto Rico Medical Center.

\begin{tabular}{lccccc}
\hline $\begin{array}{l}\text { Puerto Rico } \\
\text { Medical Center }\end{array}$ & \multicolumn{2}{c}{2003} & \multicolumn{2}{c}{2009} \\
Total No. & \multicolumn{2}{c}{ Total No. } & $P$ value \\
& M $\beta \mathrm{R}^{\mathrm{a}}$ & $\mathrm{KPC}$ & $\mathrm{M} \beta \mathrm{R}$ & $\mathrm{KPC}$ & \\
\hline MC1 & 13 & 5 & 47 & 41 & $\leq 0.01$ \\
MC2 & 16 & 2 & 36 & 27 & $\leq 0.01$ \\
MC3 & 12 & 3 & 6 & 5 & $\leq 0.01$ \\
MC4 & 11 & 1 & 20 & 19 & $\leq 0.01$ \\
\hline Total & 52 & 11 & 109 & 92 & $\leq 0.01$ \\
\hline
\end{tabular}

${ }^{\mathrm{a}} \mathrm{M} \beta \mathrm{R}$ : multi-beta-lactam resistant.

harbored either the KPC-3 or -4 variant. These results may be explained on the basis of several interacting bacterial factors, such as (1) intrinsic hydrolytic activities of the different KPC variants, (2) the genetic background and control of carbapenemase production, and (3) the presence or absence of other broad-spectrum beta-lactamases or porin mutations $[11,14,26,27,29-31]$. Although the number of isolates is small, our data is in agreement with previous reports which suggest that a $K$. pneumoniae resistant to ertapenem should alert the laboratory staff and the clinician of the possible presence of a KPC-positive isolate and the need for further testing $[2,17,32]$.

The PFGE data demonstrated a combination of bla $a_{\mathrm{KPC}}$ movements among different strains, as well as the spread of specific related groups to different sites. This implies that one or more of the following mechanisms may be responsible for the rapid intra- and interhospital dissemination of these isolates: (1) colonized human contacts (health care professionals, patients, and other individuals), (2) fomites or medical equipment as a vehicle for transmission, (3) the extensive use of broad-spectrum antibiotics creating selective pressure, and (4) the presence of the bla $a_{\mathrm{KPC}}$ within mobile genetic elements. The comparative six month data for the years 2003 and 2009 demonstrates a significant increase in the relative and absolute numbers of KPC-positive K. pneumoniae for all 4 hospitals. This may represent a combination of multiple factors such as, among others, a failure of either the hospital personnel and/or medical staff to follow the established infection control policies, increase in personnel and medical staff transit between hospitals, inability to identify an isolate promptly admitted patients colonized or infected with multidrug-resistant organisms acquired in other institutions, lack of an effective alert system between the laboratory, hospitals infection control programs, and/or the inappropriate long standing use of broad-spectrum antibiotics. The present study does not identify the relative importance of these factors or the impact of understaffing common to all hospitals. It is imperative that hospitals strictly enforce the infection control guidelines, appropriate antibiotic utilization practices, and the Center for Disease Control and Prevention recommendations for the detection and control of carbapenem-resistant Gram-negative bacilli to reduce the spread of these isolates as they have been associated with significant mortality [33-35]. 


\section{Conflict of Interests}

Dr. G. J. Vázquez reported payments for lectures from Pfizer Inc., and Merck Sharp and Dohme, Inc., research funds from Merck Sharp and Dohme, Inc. and travel funds to attend ICAAC 2010 meeting from Ortho McNeil Inc. Dr. K. S. Thomson received payments for lectures from Merck Sharp and Dohme, Inc., and research funds from Merck Sharp and Dohme, Inc. Dr. N. D. Hanson received research funds or has grants pending from Merck Sharp and Dohme, Inc. E. S. Moland and Drs. I. E. Robledo, E. E. Aquino, R. V. Goering, and M. I. Santé reported no conflict of interests relevant to this paper. I. E. Robledo and G. J. Vázquez have Joint first authorship.

\section{Acknowledgments}

This work was supported by the National Institute of Health, National Institute of General Medical Sciences, Minority Biomedical Research Program-Support for Competitive Research Enhancement (Grant no. SO6 GM008224), Research Centers in Minority Institutions (Award G12RR03051) of the National Center for Research Resources, National Institute of Health, and Merck Sharp and Dohme, Inc. The authors appreciate the support and/or technical assistance of Isabel I. Matos, Sylvia Gutierrez, Myriam Corazón, Cynthia Rivera, Pedro Rivera, Emilee Colón, Olgary Figueroa, Johanie Ortiz, Marilia Torres, Natalia Vega, Suzzette Hernández, Roberto Fernández, Xiodenis Guzmán, Caleb Fernández, Marixa Maldonado, Jesús Muñiz, María Báez, María del Mar Landrón, Jesmarie Correa, Teresa Martínez, Yolianne Rojas, Keishla González, Charlotte Torres, Jennifer Black, Jessica Lee, and Scott Andrews. They are grateful to Wieslaw Kozek and Jorge L. Santana for reviewing this paper. This work was partially presented as an oral presentation (C2-3734) at the 48th Interscience Conference on Antimicrobial Agents and Chemotherapy (ICAAC), October 25 to 26, 2008, Washington, DC, USA I. E. Robledo, by G. J. Vázquez, E. E. Aquino, E. S. Moland, M. I. Sant, and N. D. Hanson.

\section{References}

[1] K. Bush, "Alarming $\beta$-lactamase-mediated resistance in multidrug-resistant Enterobacteriaceae," Current Opinion in Microbiology, vol. 13, no. 5, pp. 558-564, 2010.

[2] P. Nordmann, G. Cuzon, and T. Naas, "The real threat of Klebsiella pneumoniae carbapenemase-producing bacteria," The Lancet Infectious Diseases, vol. 9, no. 4, pp. 228-236, 2009.

[3] A. M. Queenan and K. Bush, "Carbapenemases: the versatile $\beta$-lactamases," Clinical Microbiology Reviews, vol. 20, no. 3, pp. 440-458, 2007.

[4] P. E. Akpaka, W. H. Swanston, H. N. Ihemere et al., "Emergence of KPC-producing Pseudomonas aeruginosa in Trinidad and Tobago," Journal of Clinical Microbiology, vol. 47, no. 8, pp. 2670-2671, 2009.

[5] L. Poirel, P. Nordmann, E. Lagrutta, T. Cleary, and L. S. Munoz-Price, "Emergence of KPC-producing Pseudomonas aeruginosa in the United States," Antimicrobial Agents and Chemotherapy, vol. 54, no. 7, p. 3072, 2010.
[6] I. E. Robledo, E. E. Aquino, M. I. Sante et al., "Detection of KPC in Acinetobacter spp. in Puerto Rico," Antimicrobial Agents and Chemotherapy, vol. 54, no. 3, pp. 1354-1357, 2010.

[7] I. E. Robledo, E. E. Aquino, and G. J. Vazquez, "Detection of the KPC gene in Escherichia coli, Klebsiella pneumoniae, Pseudomonas aeruginosa, and Acinetobacter baumannii during a PCR-based nosocomial surveillance study in Puerto Rico," Antimicrobial Agents and Chemotherapy, vol. 55, no. 6, pp. 2968-2970, 2011.

[8] M. V. Villegas, K. Lolans, A. Correa, J. N. Kattan, J. A. Lopez, and J. P. Quinn, "First identification of Pseudomonas aeruginosa isolates producing a KPC-type carbapenem-hydrolyzing $\beta$-lactamase," Antimicrobial Agents and Chemotherapy, vol. 51, no. 4, pp. 1553-1555, 2007.

[9] D. J. Wolter, N. Khalaf, I. E. Robledo et al., "Surveillance of carbapenem-resistant Pseudomonas aeruginosa isolates from Puerto Rican medical center hospitals: dissemination of KPC and IMP-18 $\beta$-lactamases," Antimicrobial Agents and Chemotherapy, vol. 53, no. 4, pp. 1660-1664, 2009.

[10] C. J. Gregory, E. Llata, N. Stine et al., "Outbreak of carbapenem-resistant Klebsiella pneumoniae in Puerto Rico associated with a novel carbapenemase variant," Infection Control and Hospital Epidemiology, vol. 31, no. 5, pp. 476-484, 2010.

[11] N. Kassis-Chikhani, D. Decre, P. Ichai et al., "Outbreak of Klebsiella pneumoniae producing KPC-2 and SHV-12 in a French hospital," Journal of Antimicrobial Chemotherapy, vol. 65, no. 7, pp. 1539-1540, 2010.

[12] K. Kontopoulou, E. Protonotariou, K. Vasilakos et al., "Hospital outbreak caused by Klebsiella pneumoniae producing KPC-2 $\beta$-lactamase resistant to colistin,” Journal of Hospital Infection, vol. 76, no. 1, pp. 70-73, 2010.

[13] R. Zhang, X. D. Wang, J. C. Cai et al., "Outbreak of KPC-2producing Klebsiella pneumoniae with high qnr prevalence in a Chinese hospital," Journal of Medical Microbiology, vol. 60, no. 11, part 7, pp. 977-982, 2011.

[14] A. Zioga, V. Miriagou, E. Tzelepi et al., "The ongoing challenge of acquired carbapenemases: a hospital outbreak of Klebsiella pneumoniae simultaneously producing VIM-1 and KPC-2," International Journal of Antimicrobial Agents, vol. 36, no. 2, pp. 190-191, 2010.

[15] T. Curiao, M. I. Morosini, P. Ruiz-Garbajosa et al., "Emergence of bla KPC-3-Tn4401a associated with a pKPN3/4-like plasmid within ST384 and ST388 Klebsiella pneumoniae clones in Spain," Journal of Antimicrobial Chemotherapy, vol. 65, no. 8, pp. 1608-1614, 2010.

[16] T. Naas, G. Cuzon, M. V. Villegas, M. F. Lartigue, J. P. Quinn, and P. Nordmann, "Genetic structures at the origin of acquisition of the $\beta$-lactamase bla KPC gene," Antimicrobial Agents and Chemotherapy, vol. 52, no. 4, pp. 1257-1263, 2008.

[17] K. F. Anderson, D. R. Lonsway, J. K. Rasheed et al., "Evaluation of methods to identify the Klebsiella pneumoniae carbapenemase in Enterobacteriaceae," Journal of Clinical Microbiology, vol. 45, no. 8, pp. 2723-2725, 2007.

[18] Clinical and Laboratory Standards Institute, Performance Standards for Antimicrobial Susceptibility Testing; Twentieth Informational Supplement, vol. 30, Clinical and Laboratory Standards Institute, Wayne, Pa, USA, 2010, Vol M100-S20.

[19] G. J. Vazquez, I. E. Robledo, A. Arroyo et al., "A comparison of the antimicrobial resistance patterns of gram-negative bacilli isolated from community-private and university-affiliated hospitals from Puerto Rico," Puerto Rico Health Sciences Journal, vol. 22, no. 3, pp. 265-271, 2003. 
[20] E. S. Moland, N. D. Hanson, J. A. Black, A. Hossain, W. Song, and K. S. Thomson, "Prevalence of newer $\beta$-lactamases in gram-negative clinical isolates collected in the United States from 2001 to 2002," Journal of Clinical Microbiology, vol. 44, no. 9, pp. 3318-3324, 2006.

[21] F. J. Perez-Perez and N. D. Hanson, "Detection of plasmidmediated AmpC $\beta$-lactamase genes in clinical isolates by using multiplex PCR," Journal of Clinical Microbiology, vol. 40, no. 6, pp. 2153-2162, 2002.

[22] J. D. Pitout, A. Hossain, and N. D. Hanson, "Phenotypic and molecular detection of CTX-M- $\beta$-lactamases produced by Escherichia coli and Klebsiella spp," Journal of Clinical Microbiology, vol. 42, no. 12, pp. 5715-5721, 2004.

[23] C. C. Sanders, W. E. Sanders Jr., and E. S. Moland, "Characterization of $\beta$-lactamases in situ on polyacrylamide gels," Antimicrobial Agents and Chemotherapy, vol. 30, no. 6, pp. 951-952, 1986.

[24] R. V. Goering, "Pulsed field gel electrophoresis: a review of application and interpretation in the molecular epidemiology of infectious disease," Infection, Genetics and Evolution, vol. 10, no. 7, pp. 866-875, 2010.

[25] R. V. Goering, "Pulsed-field gel electrophoresis," in Molecular Microbiology: Diagnostic Principles and Practice, D. H. Persing, F. C. Tenover, J. Versalovic et al., Eds., pp. 185-196, ASM Press, Washington, DC, USA, 2004.

[26] D. J. Wolter, P. M. Kurpiel, N. Woodford, M. F. Palepou, R. V. Goering, and N. D. Hanson, "Phenotypic and enzymatic comparative analysis of the novel KPC variant KPC-5 and its evolutionary variants, KPC-2 and KPC-4," Antimicrobial Agents and Chemotherapy, vol. 53, no. 2, pp. 557-562, 2009.

[27] E. S. Moland, G. H. Seong, K. S. Thomson, D. H. Larone, and N. D. Hanson, "Klebsiella pneumoniae isolate producing at least eight different $\beta$-lactamases, including AmpC and KPC $\beta$-lactamases," Antimicrobial Agents and Chemotherapy, vol. 51, no. 2, pp. 800-801, 2007.

[28] M. Castanheira, H. S. Sader, and R. N. Jones, "Antimicrobial susceptibility patterns of KPC-producing or CTX-Mproducing Enterobacteriaceae," Microbial Drug Resistance, vol. 16, no. 1, pp. 61-65, 2010.

[29] J. Alba, Y. Ishii, K. Thomson, E. S. Moland, and K. Yamaguchi, "Kinetics study of KPC-3, a plasmid-encoded class A carbapenem-hydrolyzing $\beta$-lactamase," Antimicrobial Agents and Chemotherapy, vol. 49, no. 11, pp. 4760-4762, 2005.

[30] B. Kitchel, J. K. Rasheed, A. Endimiani et al., "Genetic factors associated with elevated carbapenem resistance in KPCproducing Klebsiella pneumoniae," Antimicrobial Agents and Chemotherapy, vol. 54, no. 10, pp. 4201-4207, 2010.

[31] A. L. Roth, P. M. Kurpiel, P. D. Lister, and N. D. Hanson, "blaKPC RNA expression correlates with two transcriptional start sites but not always with gene copy number in four genera of gram-negative pathogens," Antimicrobial Agents and Chemotherapy, vol. 55, no. 8, pp. 3936-3938, 2011.

[32] A. Endimiani, F. Perez, S. Bajaksouzian et al., "Evaluation of updated interpretative criteria for categorizing Klebsiella pneumoniae with reduced carbapenem susceptibility," Journal of Clinical Microbiology, vol. 48, no. 12, pp. 4417-4425, 2010.

[33] Centers for Disease Control and Prevention (CDC), "Guidance for control of infections with carbapenem-resistant or carbapenemase-producing Enterobacteriaceae in acute care facilities," Morbidity and Mortality Weekly Report, vol. 58, no. 10, pp. 256-260, 2009.
[34] S. A. Clock, B. Cohen, M. Behta, B. Ross, and E. L. Larson, "Contact precautions for multidrug-resistant organisms: current recommendations and actual practice," American Journal of Infection Control, vol. 38, no. 2, pp. 105-111, 2010.

[35] S. J. Patel, A. Oshodi, P. Prasad et al., "Antibiotic use in neonatal intensive care units and adherence with centers for disease control and prevention 12 step campaign to prevent antimicrobial resistance," Pediatric Infectious Disease Journal, vol. 28, no. 12, pp. 1047-1051, 2009. 


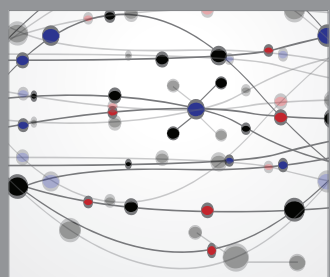

The Scientific World Journal
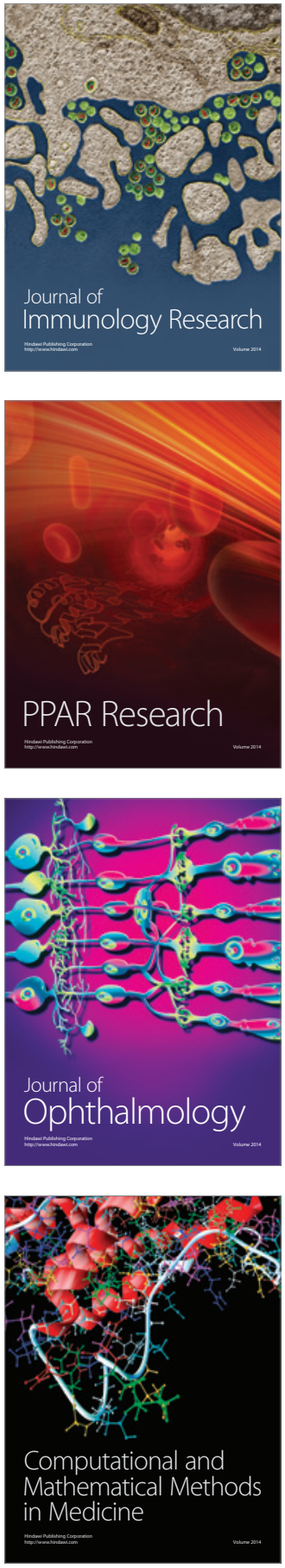

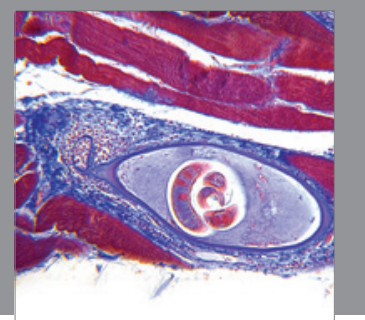

Gastroenterology

Research and Practice
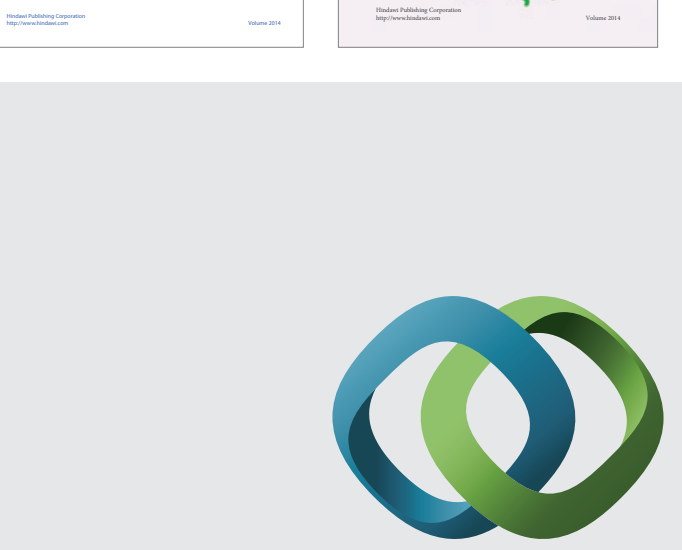

\section{Hindawi}

Submit your manuscripts at

http://www.hindawi.com
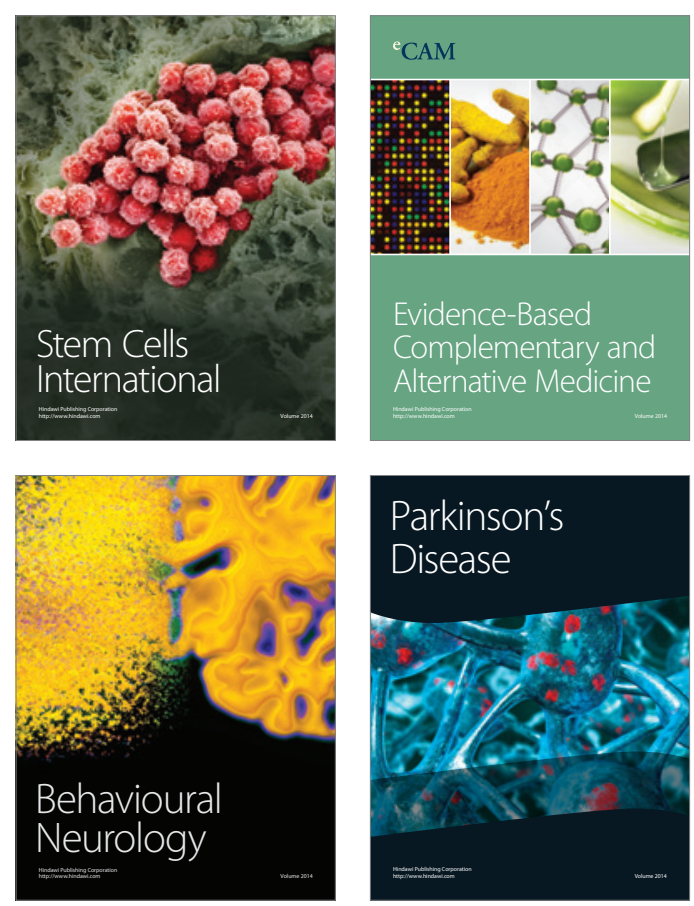

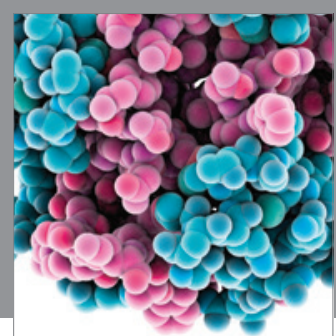

Journal of
Diabetes Research

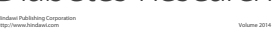

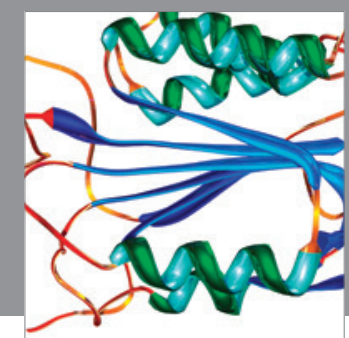

Disease Markers
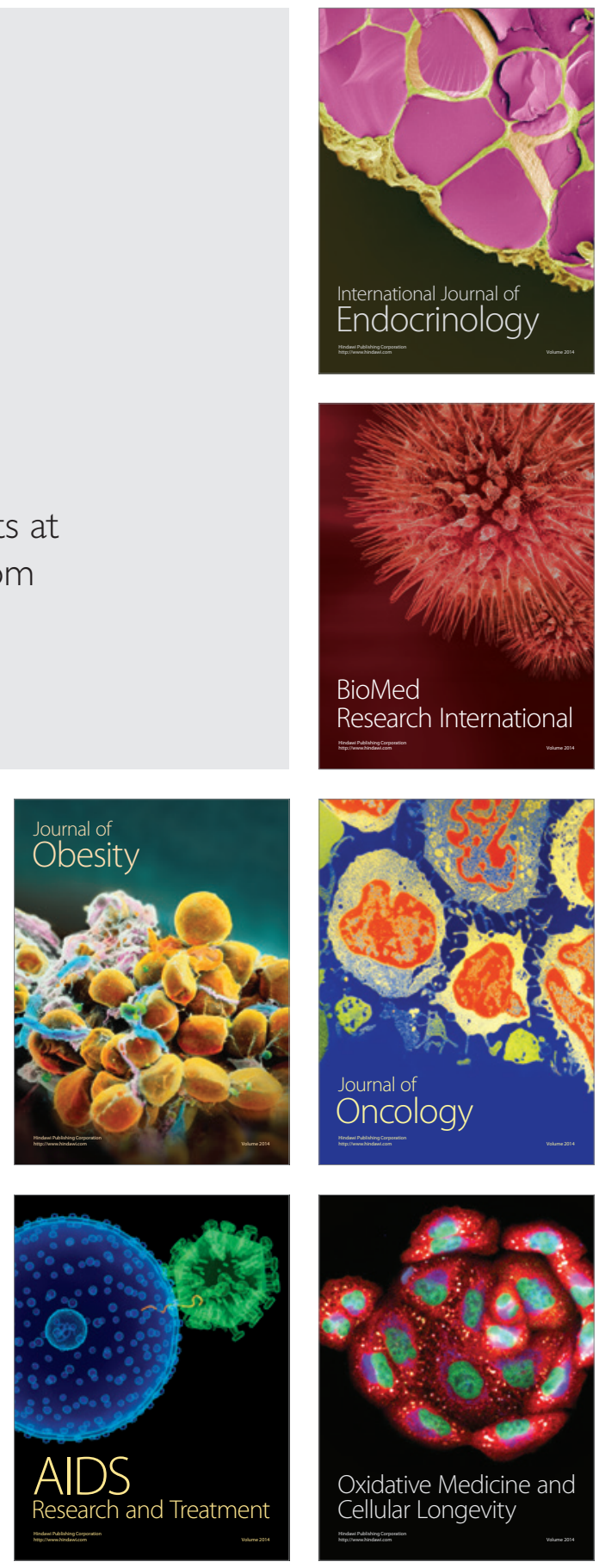